\section{Neoplasias Associadas à Síndrome dos Ovários Policísticos}

\section{RESUMO}

A síndrome dos ovários policísticos (PCOS) é a endocrinopatia mais comum em mulheres em idade reprodutiva, caracterizada pela presença de anovulação, infertilidade e hiperandrogenismo, e freqüentemente associada à obesidade e resistência insulínica. Postula-se que, a longo prazo, estas pacientes possam apresentar maior risco de neoplasias do trato reprodutivo como carcinoma (CA) de endométrio, mama e ovário. Um risco aumentado de hiperplasia e CA endometrial nessas pacientes tem sido demonstrado em vários estudos, embora seja reconhecido que a variabilidade dos critérios de seleção para o diagnóstico de PCOS em alguns destes estudos limite o valor dos dados. Apesar das pacientes com PCOS apresentarem características clínicas associadas com um aumento de risco de CA de mama, até o momento não foi possível relacionar com certeza a presença da síndrome per se com maior prevalência desta neoplasia. Finalmente, quanto ao CA do ovário, considera-se que altas concentrações locais de hormônios esteróides e fatores de crescimento representam fatores de risco para esta neoplasia. Apesar destas alterações serem comumente observadas nas pacientes PCOS não tratadas ou em tratamento para infertilidade, ainda são poucos os estudos que avaliam uma possivel relação entre PCOS e CA de ovário, mas seus resultados, embora conflitantes, sugerem ausência de associação. (Arq Bras Endocrinol Metab 2005;49/5:805-810)

Descritores: Síndrome dos ovários policísticos; Anovulação; Câncer; Endométrio; Mama; Ovários

\begin{abstract}
Polycystic Ovary Syndrome Associated Neoplasms.

Polycystic ovary syndrome (PCOS) is the most common endocrine disease in women on reproductive age. PCOS is characterized by the presence of anovulation, infertility and hyperandrogenism and is associated with obesity and insulin resistance. A major risk for neoplasms of the reproductive tract, like endometrial, breast and ovary cancer seems to be related to PCOS. While several studies have shown an increased risk for endometrial hyperplasia and cancer in PCOS patients, the variability of the selection criteria for PCOS has been recognized as a potential bias for these data. PCOS women also present clinical characteristics that are related to risk factors for breast cancer and some epidemiological evidences have been described on this issue. However, until now, a clear association between the presence of PCOS and breast carcinoma has yet not been found. Finally, high local steroid and growth factor concentrations are considered risk factors for ovary carcinoma, and are frequently observed in PCOS women. In turn, few studies have addressed the possibility of a link between PCOS and ovarian cancer and the results are conflicting but suggest that this association is unlikely. (Arq Bras Endocrinol Metab 2005;49/5:805-810)
\end{abstract}

Keywords: Polycystic ovary syndrome; Anovulation; Cancer; Endometri-

\section{revisão}

\author{
Poli Mara Spritzer \\ Débora Martinbo Morsch \\ Denusa Wiltgen
}

Unidade de Endocrinologia

Ginecológica, Serviço de

Endocrinologia, Hospital de

Clinicas de Porto Alegre;

Programa de Pós-Graduação em Ciências Médicas: Endocrinologia, Faculdade de Medicina e

Departamento de Fisiologia,

ICBS, Universidade Federal do

Rio Grande do Sul, Porto Alegre, RS. 


\section{um; Breast; Ovary}

A Síndrome dos ovÁrios policísticos (PCOS) é uma das endocrinopatias mais prevalentes, acometendo em torno de 5 a $10 \%$ das mulheres em idade reprodutiva. A PCOS caracteriza-se pela presença de anovulação, infertilidade e hiperandrogenismo, e está freqüentemente associada com obesidade e resistência insulínica, bem como com outros fatores associados à síndrome metabólica, como dislipidemia, hipertensão e diabete.

A etiopatogênese da PCOS ainda não foi esclarecida mas as evidências de inúmeros estudos indicam seu caráter multifatorial com suscetibilidade individual determinada por fatores genéticos e ambientais.

Além de maior incidência de co-morbidades metabólicas, pacientes com PCOS apresentam, a longo prazo, maior risco para hiperplasia e câncer endometrial em virtude da anovulação crônica e conseqüente estímulo estrogênico acíclico e sem oposição pela progesterona. Isto é particularmente importante em mulheres obesas, com níveis circulantes de estradiol livre mais elevados (1-3). Além disso, outras neoplasias do trato reprodutivo, dependentes de hormônios sexuais, têm sido relacionadas com PCOS, em especial o carcinoma de mama e de ovário.

Entretanto, por sua natureza complexa e apresentação clínica heterogênea, torna-se difícil definir que fatores relacionados com a PCOS - se a hiperinsulinemia, a concentração elevada de fatores de crescimento, LH ou androgênios, a obesidade, ou fatores genéticos - representam maior risco para o desenvolvimento de câncer.

\section{Câncer de endométrio}

O carcinoma endometrial é o câncer mais comum nos Estados Unidos da América (EUA) e, na Europa, foi descrita uma proporção de mais de 1 caso de câncer de endométrio para cada vinte casos de câncer feminino $(4,5)$. A média de idade por ocasião do diagnóstico é de 61 anos; contudo, em 5 a 30\% das mulheres o diagnóstico é realizado antes dos 50 anos (4). Soliman e col. (4) estudaram mulheres pré-menopáusicas com menos de 50 anos de idade apresentando adenocarcinoma endometrial; observaram que $58 \%$ das pacientes tinham um índice de massa corporal (IMC) maior ou igual a $30 \mathrm{~kg} / \mathrm{m}^{2}, 55 \%$ eram nulíparas e $39 \%$ relatavam ciclos menstruais irregulares. A incidência de diabetes e hipertensão foi de $23 \%$.

Embora a freqüência de câncer endometrial entre mulheres pré e peri-menopáusicas venha diminuindo, em alguns países europeus parece haver uma tendência a maior incidência de casos entre mulheres na pós-menopausa (5). Mudanças no comportamento reprodutivo com decréscimo na fertilidade no menacme e a prevalência de sobrepeso e obesidade podem explicar parcialmente estas observações.

São considerados fatores de risco para o carcinoma de endométrio as terapias estrogênicas prolongadas sem oposição por progestogênios, os tumores produtores de estrogênios, obesidade, nuliparidade (6), ciclos menstruais irregulares $(7,8)$, menarca precoce, menopausa tardia (9), diabetes mellitus e hipertensão arterial $(10,11)$. Neste sentido, não é surpreendente que o carcinoma de endométrio seja mais freqüente nos países desenvolvidos, uma vez que se associa mais ao estilo de vida ocidental (obesidade e sedentarismo) do que a fatores genéticos.

Embora apenas nas últimas décadas tenha sido reconhecido que os fatores de risco para carcinoma de endométrio são também mais freqüentes em pacientes com PCOS, a associação entre PCOS e câncer endometrial vem sendo sugerida desde 1949 (12). Inúmeros estudos reforçam esta idéia, como o de Coulam e col. (1), que observaram um risco de 3,1 (CI 96\% 1,1 $7,3)$ para desenvolver câncer de endométrio em 1.270 pacientes com anovulação crônica.

Nas pacientes com PCOS, os androgênios, principalmente a androstenediona, são a principal fonte de estrogênios. $\mathrm{O}$ excesso de androgênios, especialmente se associado à obesidade, reduz a síntese hepática da globulina ligadora de hormônios esteróides (SHBG), aumentando assim as concentrações circulantes de androgênios livres. A obesidade promove ainda um estímulo adicional sobre a conversão periférica de androgênios em estrogênios. A anovulação crônica associada ao hiperandrogenismo perpetua a secreção continuada de estrogênios e a deficiência de progesterona, contribuindo para um maior risco de câncer de endométrio $(13,14)$. A hiperinsulinemia pode também exercer influência sobre o risco para o carcinoma endometrial, e os mecanismos envolvidos estariam associados à redução da SHBG circulante e/ou elevação dos níveis séricos de IGFl e estrogênios (13-18).

Além disso, foram identificados sítios de ligação para insulina no endométrio de mulheres normais e pacientes com câncer endometrial, e, nestas últimas, concentrações elevadas de insulina plasmática foram também detectadas $(19,20)$. Níveis circulantes aumentados de insulina, como é com freqüência observado na PCOS, poderiam acelerar o crescimento do câncer endometrial (21).

A hipersecreção de LH, alteração hormonal comum nestas pacientes, tem sido igualmente implica- 
da no desenvolvimento do câncer endometrial em mulheres com PCOS $(14,22,23)$. Konishi e col. (23) demonstraram um aumento na expressão gênica e protéica de receptores para LH e gonadotrofina coriônica humana (hCG) em biópsias de hiperplasia e de câncer endometrial, que se desenvolvem em mulheres jovens anovulatórias, incluindo-se aquelas com PCOS.

A hiperplasia endometrial pode apresentar-se como precursora do câncer endometrial. É mais freqüentemente diagnosticada em mulheres na pósmenopausa, mas mulheres de qualquer idade com secreção estrogênica não oposta por progesterona encontram-se em situação de risco aumentado para o desenvolvimento da hiperplasia endometrial (24). Ho e col. (25) demonstraram que a incidência de carcinoma de endométrio seguindo a hiperplasia endometrial foi de $27,6 \%$ na presença de atipia e $3,4 \%$ nas pacientes sem atipia. Subfertilidade e PCOS foram mais prevalentes nos casos com atipia, mas não entre os casos de carcinoma. Androgênios circulantes elevados também têm sido associados com a hiperplasia de endométrio, a qual geralmente precede e acompanha a ocorrência de carcinoma endometrial (26). Mais recentemente, Ricci e col. (27) demonstraram, através de um estudo caso-controle envolvendo 1.029 mulheres italianas com hiperplasia endometrial, que o nível de educação formal, obesidade, diabete e uso de terapia hormonal aumentam o risco de hiperplasia endometrial.

No entanto, é importante salientar que o conjunto de evidências para a associação entre câncer endometrial e PCOS esbarra em algumas limitações. As mais importantes são, de um lado, o pequeno número de casos estudados de carcinoma endometrial em pacientes com PCOS e, de outro, possíveis vieses de seleção relacionados com a ampla variabilidade de critérios utilizados por diferentes autores para definir PCOS até recentemente. Além disso, a ausência de grupo-controle em muitos dos estudos publicados dificulta a realização de meta-análises que pudessem calcular um risco relativo estimado para o carcinoma endometrial em mulheres com PCOS. Recentemente, Furberg \& Thune (11), estudando mulheres obesas com carcinoma endometrial, mostraram um aumento de risco de 2,6 a 3 vezes comparadas com pacientes não obesas. Assim, é possível que alguns estudos se refiram, na verdade, a casos inespecíficos de infertilidade e/ou obesidade, e que os dados disponíveis não sejam suficientemente robustos para definir o risco real para o desenvolvimento de câncer endometrial em pacientes com PCOS $(3,14,21,28)$. Contudo, apesar da impossibilidade atual de definir risco, é consensual que pacientes com PCOS, em especial aquelas com oligo/amenorréia, recebam medidas de prevenção para hiperplasia endometrial, como ciclos menstruais induzidos e controle ecográfico periódico da espessura endometrial.

\section{Câncer de mama}

O câncer de mama é a neoplasia mais comum em mulheres, representando $22 \%$ de todos os carcinomas diagnosticados em 2000 nos EUA, e a segunda maior causa de morte por câncer ( $15 \%$ das mortes por neoplasia) nesta população (29). Associado a fatores genéticos, estudos epidemiológicos conduzidos em diferentes populações determinaram que fatores de risco como idade, localização geográfica, status sócioeconômico e eventos reprodutivos (idade da menarca e da menopausa, infertilidade, gravidez e amamentação) estão associados à maior incidência de carcinoma (Ca) de mama (30). Outros fatores de risco também reconhecidos estão relacionados com o estilo de vida (abuso de álcool, dieta inadequada, obesidade e sedentarismo) e com concentrações circulantes de hormônios endógenos ou com o uso de hormônios exógenos (hiperandrogenismo, terapia hormonal e contraceptivos orais) (30). Contudo, apesar de pacientes com PCOS apresentarem muitos destes fatores, evidências até o momento não puderam confirmar que a presença desta síndrome esteja relacionada a uma maior incidência de Ca de mama.

Coulan e col. (1) avaliaram uma amostra de pacientes com anovulação crônica e verificaram que $o$ risco relativo relacionado à anovulação foi de $1,5(0,75-$ 2,55 , IC 95\%) na população total em estudo. Entretanto, após estratificação por idade, mulheres já no período pós-menopausa mas com história prévia de anovulação apresentaram risco relativo de 3,6 (1,2-8,7, IC 95\%) para o desenvolvimento de Ca de mama (1).

Já outros autores, em estudo de caso-controle, reportaram que a presença de PCOS poderia ser um fator protetor quanto ao surgimento de $\mathrm{Ca}$ de mama, demonstrando uma razão de chances de 0,52 (0,32 $0,87)(31)$. No entanto, uma crítica a estes estudos envolve os critérios de definição de PCOS (questionário de auto-definição pela paciente da presença ou ausência da síndrome), o que determinou uma prevalência muito baixa quando comparada com as taxas segundo os critérios diagnósticos atuais $-0,5$ a $1 \%$ contra 5 a $10 \%$ em muitas populações mundiais, respectivamente (32-34). Anderson e col. estudaram 34.835 mulheres e, apesar da baixa prevalência de $\operatorname{PCOS}(1,35 \%)$, concluíram que não há associação da síndrome com desenvolvimento de $\mathrm{Ca}$ de mama, mesmo após correção para fatores de riscos adicionais como idade, uso de anticoncepcional oral, 
obesidade central e história familiar (35).

Um outro aspecto que vem sendo examinado quanto a uma possível associação entre PCOS e Ca de mama é o risco associado ao uso de drogas indutoras da ovulação (citrato de clomifeno e gonadotrofinas), utilizadas com freqüência nas mulheres com PCOS e infertilidade. A análise conjunta dos estudos de coorte e casocontrole não demonstra clara implicação destes medicamentos e surgimento do Ca de mama (36). Porém, em uma amostra de mulheres australianas houve um aumento de duas vezes na incidência de Ca de mama durante o primeiro ano de tratamento para fertilização in vitro (37), sugerindo que drogas indutoras de superovulação possam promover rápido crescimento de tumores préexistentes, similares ao aumento transitório na incidência de Ca de mama visto logo após uma gravidez (38).

Estudo recente utilizando uma coorte de 9.087 mulheres com doenças benignas de mama demonstrou que, na presença de tipo histológico mais agressivo e de história familiar positiva, estas alterações são fatores de risco para câncer de mama (39). Contudo, no mesmo período, Soran e cols. avaliaram 240 mulheres (116 PCOS e 124 controles) que fazem parte de uma coorte agora com seguimento de 12 anos e não observaram maior prevalência de alterações benignas de mama (doença fibrocística, calcificações, fibroadenomas, eritema, secreção, hiperplasia) nas pacientes com PCOS (40). Este achado contrapõe dados prévios da literatura, os quais demonstravam associação entre morfologia policística ovariana e/ou PCOS e alterações mamárias benignas $(41,42)$.

Em conclusão, apesar de as pacientes com PCOS apresentarem fatores de risco para Ca de mama, até o momento não foi possível relacionar a presença da síndrome per se com maior prevalência desta neoplasia.

\section{Câncer de ovário}

$\mathrm{O}$ câncer de ovário é a sexta causa de malignidade e a quinta causa de morte em mulheres nos EUA (43), sendo em cerca de $90 \%$ dos casos do tipo histológico epitelial. Atualmente, considera-se que não há um fator de risco único implicado na etiologia deste tipo de tumor; contudo, um risco inerente para câncer de ovário parece existir naquelas pacientes com múltiplas ovulações porém nulíparas (inférteis), com menarca precoce e menopausa tardia $(28,44)$. Em estudo realizado com 197 mulheres com câncer de ovário, as pacientes nulíparas apresentaram um risco 2,45 vezes maior de desenvolver a neoplasia quando comparadas às mulheres que estiveram grávidas 3 ou mais vezes. Já mulheres que engravidaram pelo menos 1 vez apresentaram uma redução de risco de 1,27 vezes quando comparadas às nulíparas (45).

Embora pacientes com PCOS apresentem alterações consideradas como fatores de risco para $\mathrm{Ca}$ de ovário, como altas concentrações locais de hormônios esteróides e fatores de crescimento, ainda são poucos os estudos que avaliam a associação entre PCOS e câncer de ovário, e seus resultados são conflitantes. Enquanto Coulam e col. (1) não encontraram associação entre mulheres anovulatórias e aumento de risco de carcinoma ovariano, um estudo de caso-controle (46) sugere que a presença de PCOS determina um aumento de 2,5 (1,1 - 5,9, IC 95\%) no risco de desenvolver esta neoplasia. Entretanto, a validade externa destes estudos é questionável, já que a prevalência encontrada de PCOS foi muito baixa quando comparada a outras populações.

Quanto ao risco de câncer ovariano nas pacientes PCOS submetidas a tratamento com drogas indutoras de ovulação (36), os dados da literatura são ainda controversos. Em uma meta-análise, que reuniu 12 estudos na tentativa de elucidar a etiologia do câncer ovariano, demonstrou-se que o uso prévio de medicações utilizadas para induzir superovulações estava associado com uma razão de chances de 2,8 (1,3-6,1, IC 95\%) para esta neoplasia, essencialmente no grupo das nulíparas (47). Em outro estudo de coorte retrospectiva com 3.837 mulheres avaliadas para infertilidade na cidade de Seattle entre os anos de 1974 e 1985, o uso de citrato de clomifeno esteve associado com um risco ajustado de 2,3 vezes maior para câncer de ovário (48). Contudo, estudos mais recentes e metodologicamente mais adequados não reproduziram estes achados de aumento de risco relacionado à utilização de citrato de clomifeno ou gonadotrofinas $(49,50)$. Assim, as evidências atuais não permitem afirmar de forma conclusiva que haja associação entre uso de drogas para indução de ovulação e câncer ovariano. Entretanto, como a maioria dos estudos foi realizada com amostras pequenas e pouco tempo de seguimento, estudos adicionais devem monitorar os efeitos a longo prazo, com atenção especial às mulheres que persistirem nulíparas, dentre elas as pacientes com PCOS.

\section{REFERÊNCIAS}

1. Coulam CB, Annegers JF, Kranz JS. Chronic anovulation syndrome and associated neoplasia. Obstet Gynecol 1983;61:403-7.

2. Key TJA, Pike MC. The dose-effect relationship between "unoppposed" oestrogens and endometrial mitotic rate: its central role in explaining and predicting 
endometrial cancer risk. Br J Cancer 1988;57:205-12.

3. Solomon CG. The epidemiology of polycystic ovary syndrome. Prevalence and associated disease risks. Endocrinol Metab Clin North Am 1999;28(2):247-63.

4. Soliman PT, Oh JC, Schmeler KM, Sun CC, Slomovitz BM, Gershenson DM, et al. Risk factors for young premenopausal women with endometrial cancer. Obstet Gynecol 2005;105(3):575-80.

5. Bray F, dos Santos Silva I, Moller H, Weiderpass E. Endometrial cancer incidence trends in Europe: underlying determinants and prospects for prevention. Cancer Epidemiol Biomarkers Prev 2005; 14(5): 1 132-42.

6. Niwa K, Imai A, Hashimoto M, Yokoyama Y, Mori H, Matsuda $Y$, et al. A case-control study of uterine endometrial cancer of pre- and post-menopausal women. Oncol Rep 2000;7(1):89-93.

7. Henderson BE, Casagrande JT, Pike MC. The epidemiology of endometrial cancer in young women. Br J Cancer 1983;47:749-56.

8. Dahlgren E, Friberg LG, Johansson S. Endometrial carcinoma: ovarian dysfunction - a risk factor in young women. J Obstet Gynecol Reprod Biol 1991;41:143-50.

9. Elwood JM, Cole P, Rothman KJ. Epidemiology of endometrial cancer. J Natl Cancer Inst 1977;59:105560.

10. Gordon J, Reagan JW, Finkle WD, Ziel HK. Estrogen and endometrial carcinoma. An independent pathology review supporting original risk estimate. N Engl J Med 1977;297:570-1.

11. Furberg AS, Thune I. Metabolic abnormalities (hypertension, hyperglycemia and overweight), lifestyle (high energy intake and physical inactivity) and endometrial cancer risk in a Norwegian cohort. Int J Cancer 2003; 104:669-76.

12. Speert $H$. Carcinoma of the endometrium in young women. Surg Gynaecol Obstet 1949;88:332-6.

13. Meirow D, Schenker JG. The link between female infertility and cancer: epidemiology and possible aetiologies. Human Reproduction Update 1996;2(1):63-75.

14. Gadducci A, Gargini A, Palla E, Fanucchi A, Genazzani AR. Polycystic ovary syndrome and gynecological cancers: is there a link? Gynecol Endocrinol 2005; 20(4):200-8.

15. Gibson M. Reproductive health and polycystic ovary syndrome. Am J Med 1995;98(1A):67S-75S.

16. Pasquali R, Vicennati V, Bertazzo D, Casimirri F, Pascal $G$, Tortelli $O$, et al. Determinants of sex hormone-binding globulin blood concentrations in premenopausal and postmenopausal women with different estrogen status. Virgilio-Menopause-Health Group. Metabolism 1997; 46:5-9.

17. Pugeat M, Crave JC, Elmidani M, Nicolas MH, Garoscio $\mathrm{CM}$, Lejeune $\mathrm{H}$, et al. Pathophysiology of sex hormone binding globulin (SHBG): relation to insulin. J Steroid Biochem Mol Biol 1991;40:841-9.

18. Crave JC, Fimbel S, Lejeune H, Cugnardey N, Dechaud $\mathrm{H}$, Pugeat $\mathrm{M}$. Effects of diet and metformin administration on sex hormone-binding globulin, androgens, and insulin in hirsute and obese women. J Clin Endocrinol Metab 1995;80:2057-62.

19. Nagamani M, Stuart CA, Dunhardt PA, Doherty PA. Specific binding sites for insulin and insulin-like growth factor I in human endometrial cancer. Am J Obstet Gynecol 1991;165:1865-71.

20. Bershtein LM, Gamaiunova VB, Kvachevskaia luO, Tsyrlina EV, Kovalenko IG. The nature of hyperinsulinaemia (insulin resistance) in endometrial carcinoma: of plasma levels of insulin and c-peptide. Vopr Onkol 2000,46:1915.

21. Hardiman P, Pillay OS, Atiomo W. Polycystic ovary syndrome and endometrial carcinoma. Lancet 2003;361: 1810-2.

22. Lincoln SR, Lei ZM, Ackermann DM. The expression of human chorionic gonadotrophin/human luteinizing hormone receptors in human endometrial and myometrial blood vessels. J Clin Endocrinol Metab 2002;76:1 140-4.

23. Konishi I, Koshiyama M, Mandai M, Kuroda $H$, Yamamoto S, Nanbu K, et al. Increased expression of LH/hCG receptors in endometrial hyperplasia and carcinoma in anovulatory women. Gynecol Oncol 1997;65:273-80.

24. Montgomery BE, Daum GS, Dunton CJ. Endometrial hyperplasia: a review. Obstet Gynecol Surv 2004;59(5): 368-78.

25. Ho SP, Tan KT, Pang MW, Ho TH. Endometrial hyperplasia and the risk of endometrial carcinoma. Singapore Med J 1997;38(1):11-5.

26. Vitoratos N, Gregoriou O, Hassiakos D, Zourlas PA. The role of androgens in the late-premenopausal woman with adenomatous hyperplasia of the endometrium. Int J Gynaecol Obstet 1991;34:157-61.

27. Ricci E, Moroni S, Parazzini F, Surace M, Benzi G, Salerio $B$, et al. Risk factors for endometrial hyperplasia: Results from a case-control study. Int J Gynecol Cancer 2002; 12:257-60.

28. Balen A. Polycistic ovary syndrome and cancer. Hum Reprod Update 2001;7:522-5.

29. Parkin DM. International variation. Oncogene 2004;23: 6329-40.

30. Dumitresco RG, Cotarla I. Understanding breast cancer risk - where do we stand in 2005? J Cell Mol Med 2005;9(1):208-21.

31. Gammon MD, Thompson WD. Polycystic ovaries and the risk of breast cancer. Am J Epidemiol 1991;134:81824.

32. Ehrmann DA, Barnes RB, Rosenfield RL. Polycystic ovary syndrome as a form of functional ovarian hyperandrogenism due to disregulation of androgen secretion. Endocr Rev 1995;16:322-53.

33. Knochenhauer ES, Key TJ, Kahsar-Miller M, Waggoner W, Boots LR, Azziz R. Prevalence of the polycystic ovary syndrome in unselected black and white women of the southeastern United States: a prospective study. J Clin Endocrinol Metab 1998;83:3078-82.

34. Asuncion M, Calvo RM, San Millan JL, Sancho J, Avila S, Escobar-Morreale HF. A prospective study of the prevalence of the polycystic ovary syndrome in unselected 
Caucasian women from Spain. J Clin Endocrinol Metab 2000;85:2434-8.

35. Anderson KE, Sellers TA, Chen PL. Association of SteinLeventhal syndrome with the incidence of postmenopausal breast carcinoma in a large prospective study of women in lowa. Cancer 1997;79:494-9.

36. Brinton LA, Moghissi KS, Scoccia B, Westhoff CL, Lamb EM. Ovulation induction and cancer risk. Fertil Steril 2005;83:261-74.

37. Venn A, Watson L, Bruinsma F, Giles G, Healy D. Risk of cancer after use of fertility drugs with in-vitro fertilization. Lancet 1999:354:1586-90.

38. Lambe M, Hsieh C, Trichopoulos D, Ekborn A, Pavia M, Adami HO. Transient increase in the risk of breast cancer after giving birth. N Engl J Med 1994;331:5-9.

39. Hartmann LC, Sellers TA, Frost MA, Lingle WL, Degnim $\mathrm{AC}$, Ghosh K, et al. Benign breast disease and the risk of breast cancer. N Engl J Med 2005;353:229-37.

40. Soran A, Talbott EO, Zborowski JV, Wilson JW. The prevalence of benign breast disease in women with polycystic ovary syndrome: a review of a 12-year follow-up. Int J Clin Pract 2005;59(7):795-7.

41. Anderson KE, Sellers TA, Chen PL, Rich SS, Hong CP, Folsom AR. Association of Stein-Leventhal syndrome with the incidence of postmenopausal breast carcinoma in a large prospective study of women in lowa. Cancer 1997;79(3):494-9.

42. D'Amelio R, Farris M, Grande S, Feraudo E, luliano A, Zichella $L$. Association between polycystic ovary and fibrocystic breast disease. Gynecol Obstet Invest 2001;51(2):134-7.
43. Heintz AP, Hacker NF, Lagasse LD. Epidemiology and etiology of ovarian cancer: a review. Obstet Gynecol 1985;66:127-35.

44. Meirow D, Schenker JD. The link between female infertility and cancer: epidemiology and possible aetiologies. Hum Reprod Update 1996;2:63-75.

45. McGowan L, Parent L, Lednar W, Norris HJ. The woman at risk for developing ovarian cancer. Gynecol Oncol 1979:7:325-44.

46. Schildkraut JM, Schwingl PJ, Bastos E. Epithelial ovarian cancer risk among women with polycystic ovary syndrome. Obstet Gynecol 1996;88:554-9.

47. Whittemore AS, Harris R, Itnnyre J, and the Collaborative Ovarian Cancer Group. Characteristics relating to ovarian cancer risk: collaborative analysis of 12 US casecontrol studies. Am J Epidemiol 1992;136:1 184-203.

48. Rossing MA, Daling JR, Weiss NS, Moore DE, Self SG. Ovarian tumors in a cohort of infertile women. $\mathbf{N}$ Engl $\mathbf{J}$ Med 1994;331:771-6.

49. Brinton LA, Lamb EJ, Moghissi KS, Scoccia B, Althuis MD, Mabie JE. Ovarian cancer risk after use of ovulationstimulant drugs. Obstet Gynecol 2004;103:1 194-203.

50. Croughan-Minihane MS, Camarano L, Feigenbaun S, Nelson H, Adamson GD, Cadius MM. The risk of ovarian cancer associated with infertility and infertility treatments. Fertil Steril 2001;76:68.

\section{Endereço para correspondência:}

Poli Mara Spritzer

Serviço de Endocrinologia

Hospital de Clínicas de Porto Alegre

Rua Ramiro Barcelos 2350

90050-170 Porto Alegre, RS

Fax: (51) 3316-3656

E-mail: spritzer@ufrgs.br 